\title{
IDENTIFICATION OF TRABECULAR EXCRESCENCES, NOVEL MICROANATOMICAL STRUCTURES, PRESENT IN BONE IN OSTEOARTHROPATHIES
}

\author{
AM Taylor ${ }^{1 *}$, A Boyde 2 , JS Davidson ${ }^{3}$, JC Jarvis ${ }^{4}$, LR Ranganath ${ }^{4}$ and JA Gallagher ${ }^{4}$
}

\author{
${ }^{1}$ Lancaster Medical School, Faculty of Health and Medicine, Lancaster University, Lancaster, UK. \\ ${ }^{2}$ Institute of Dentistry, Barts and The London School of Medicine and Dentistry, Queen Mary University of London, \\ London, UK.
}

${ }^{3}$ Department of Trauma and Orthopaedics, Royal Liverpool and Broadgreen University Hospitals Trust, Liverpool, UK. ${ }^{4}$ Department of Musculoskeletal Biology, Institute of Ageing and Chronic Disease, University of Liverpool, Liverpool,

UK.

\begin{abstract}
It is widely held that bone architecture is finely regulated in accordance with homeostatic requirements. Aberrant remodelling (hyperdensification and/or cyst formation in the immediately subchondral region) has previously been described in bone underlying cartilage in arthropathies. The present study examined the trabecular architecture of samples of bone, initially in the severe osteoarthropathy of alkaptonuria, but subsequently in osteoarthritis using a combination of light microscopy, 3D scanning electron microscopy and quantitative backscattered electron scanning electron microscopy. We report an extraordinary and previously unrecognised bone phenotype in both disorders, including novel microanatomical structures. The underlying subchondral trabecular bone contained idiosyncratic architecture. Trabecular surfaces had numerous outgrowths that we have termed "trabecular excrescences", of which three distinct types were recognised. The first type arose from incomplete resorption of branching secondary trabeculae arising from the deposition of immature (woven) bone in prior marrow space. These were characterised by very deeply scalloped surfaces and rugged edges. The second type had arisen in a similar way but been smoothed over by new bone deposition. The third type, which resembled coarse stucco, probably arises from resting surfaces that had been focally reactivated. These were poorly integrated with the prior trabecular wall. We propose that these distinctive microanatomical structures are indicative of abnormal osteoclast/osteoblast modelling in osteoarthropathies, possibly secondary to altered mechanical loading or other aberrant signalling. Identification of the mechanisms underlying the formation of trabecular excrescences will contribute to a better understanding of the role of aberrant bone remodelling in arthropathies and development of new therapeutic strategies.
\end{abstract}

Keywords: Bone remodelling, scanning electron microscopy, osteoarthritis, alkaptonuria.

*Address for correspondence:

Dr Adam Taylor

Faculty of Health and Medicine

C16, Faraday Building

Lancaster University, Bailrigg

Lancaster, LA1 4YB, UK

Telephone Number: +44 (0)1524 592503

FAX Number: +44 (0)1524 593747.

E-mail: a.m.taylor@lancs.ac.uk

\section{Introduction}

Turnover of skeletal tissues occurs as part of development and continues throughout life, in both health and disease. It is usually held and widely stated that skeletal architecture is finely regulated in accordance with homeostatic requirements by bone modelling (net deposition or resorption) and remodelling (resorption and subsequent deposition) and that one of the major influences on remodelling is mechanical loading. The tissue level responses at the micro and ultra-structural levels are complex chains of events involving a number of specialised layers within the joint, namely hyaline articular cartilage (HAC), articular calcified cartilage (ACC), subchondral bone (SCB), trabecular and cortical bone. The relationships between bone and cartilage are highly dependent on reciprocal normal functions of the tissues. Under normal loading, HAC transmits forces through the ACC to SCB, resulting in high shear stresses at the osteochondral junction region brought about by differences in the material properties of the calcified and non-calcified tissues (Flachsmann et al., 2000 and Ferguson et al., 2003). Remodelling of bone is usually highly regulated with a balance of bone removal and formation (Frost, 1964) in what is generally considered to be a coupled mechanism (Parfitt, 1982). However, alterations in trabecular architecture with the normal ageing process suggest that the coupling mechanism is not as tightly regulated as it appears to be in earlier life (Jayasinghe et al., 1993 and 1994).

Remodelling is regulated by a complex interaction of systemic and local factors including paracrine and autocrine signals. The topography of the surface that is produced by the osteoclasts is also important in regulating new bone formation (Gray et al., 1996). While coupling and (re)modelling is focal at multiple locations at any given time (Frost, 1991), the systemic hormones and the cells exhibit a multitude of wider reaching effects which result in the replication of undifferentiated cells, recruitment of cells and the differentiated function of cells (Hill, 1998).

Deviation from the finely balanced and highly regulated (re)modelling process results in numerous recognised pathological conditions in the mineralised tissues. These include osteoporosis, in which resorption exceeds formation (Russell, 2003). Aberrant remodelling of bone underlying cartilage is frequently observed in arthropathies, the most common of which is osteoarthritis (OA). There is still much debate about what instigates 
pathological change(s) in tissues in OA, and whether the defect occurs in the HAC or the SCB first, or whether they occur simultaneously. However, the changes in both tissues bring about altered loading across the joint tissues.

We have been investigating the severe osteoarthropathy in patients with alkaptonuria (AKU). This is a rare autosomal recessive condition that results in a devastating early onset osteoarthropathy of the weight bearing joints following presentation of radiographic and clinical symptoms analogous with OA (Bálint and Szebenyi, 2000; Lagier, 2006). Its recognition as a rare form of OA is also supported by common end point treatment of both disorders - joint replacement (Kraus, 2011). In AKU, it is commonly held that cartilage is the predominantly affected tissue, although it has been shown that capsular tissues are also involved. Ochronotic pigment derived from polymerisation of homogentisic acid (HGA) is initially associated at the ultrastructural level with the periodicity of the collagen fibrils (Taylor et al., 2010). The evidence for the association of HGA pigment with collagen is clear, but until recently it was accepted that bone is relatively unaffected, particularly compared to cartilage (Di Franco et al., 2000). We have shown that part of the progression of the destructive mechanism in AKU involves an upregulation of the osteoclastic activity in the mineralised joint tissues of these patients resulting in the unprecedented complete resorption of the SCB plate and ACC (Taylor et al., 2011). This resorption may be induced by altered loading, possibly as a result of stress shielding due to the altered mechanical properties of the overlying HAC. Ochronotic pigment deposition increases the stiffness of the HAC matrix, resulting in abnormal distribution of load through the tissues (Taylor et al., 2011). Alternatively, HGA polymer deposition may inhibit the mineralisation of HAC to become ACC and the reserve of ACC may eventually be used up.

In this study, we analysed samples from AKU arthropathies following our previous discovery of the exceptional SCB and ACC phenotype and compared them with more common OA arthropathy samples. In performing this study we were able to identify novel microanatomical structures in bone of patients with ochronotic arthropathy, which may be the result of the abnormal loading in the tissues following changes in the load distribution across the joint because of aggressive remodelling of the SCB and ACC plate. These microanatomical structures were discovered using 3 dimensional scanning electron microscopy (3D-SEM). This high depth of field technique, even when used at low magnification, allowed the identification and observation of numerous excrescences on free bone surfaces. Subsequently, conventional light microscopic histology and quantitative backscattered electron mode scanning electron microscopy (qBSE-SEM) both confirmed their presence in the calcified matrix. The number and type of excrescence were more difficult to distinguish by these latter imaging methods as they are limited either by the thickness of the specimens $-\sim 5 \mu \mathrm{m}$ for light microscopy - or by the information depth of the method $-\sim 0.5 \mu \mathrm{m}$ for $20 \mathrm{kV}$ qBSE-SEM.

Unexpectedly we subsequently observed these structures in 'ordinary' osteoarthritic samples that were being used as control, reference specimens for the investigation, and, retrospectively, in other archival OA samples used for BSE-SEM studies. These structures add evidence to the literature that already describes the abnormal structure and function of tissues in joint arthropathy.

\section{Materials and Methods}

\section{Sample acquisition}

All joint tissues were obtained under ethical approval (Liverpool REC) with informed patient consent from patients with clinically diagnosed AKU or OA undergoing joint replacement surgery for joint arthropathy. 12 AKU samples (range; $46-70$ years, mean 58 years) and 10 OA (6 of which were analysed as part of a previous microscopical study by Ferguson et al, 2003); range 55-89 years, mean 70 years and the remaining 4 were new samples; range 51-74 years, mean 62 years) were washed in phosphatebuffered saline (PBS) at time of surgery and fixed in $10 \%$ phosphate buffered formol saline. Samples were processed routinely for histology and for topographical 3D-SEM and qBSE-SEM as follows.

\section{Topographical 3D scanning electron microscopy}

Bone slices were macerated for $18 \mathrm{~d}$ in $4 \%$ Tergazyme, bacterial pronase enzyme detergent (Alconox Inc., New York, NY, USA), followed by $29 \mathrm{~d}$ in $5 \%$ hydrogen peroxide solution (i.e., 6 fold dilution of the Aristar stock concentrate) to remove all cells and normal hyaline cartilage matrix and leave all calcified matrices. After washing and drying, these slices were carbon coated from both sides and imaged using $20 \mathrm{kV}$ 3D-BSE-SEM (Figs. 1,2 and 5).

\section{Histology}

Soft tissues were dissected and then processed through graded alcohols into xylene before being embedded in paraffin. Calcified tissues were decalcified in $12 \%$ EDTA with $10 \%$ formalin before being processed through graded alcohols and xylene. Histological sections were cut at $5 \mu \mathrm{m}$ and mounted on glass slides before being deparaffinised, stained with $\mathrm{H}$ and $\mathrm{E}$, dehydrated through graded alcohols and mounted in DPX (Sigma Aldrich, Poole, UK). Direct view stereoscopic fluorescence microscopy was conducted on unstained histological sections using an Edge 3D microscope fitted with twin Canon digital cameras (Figs. 3 and 6).

\section{Quantitative back scattered electron microscopy}

Other slices were embedded in polymethylmethacrylate (PMMA), the block surfaces trimmed and polished, and coated with carbon by evaporation. They were imaged using backscattered electrons (BSE) in a Zeiss DSM962 SEM with external computer control. Images were recorded at $20 \mathrm{kV}, 0.5 \mathrm{nA}$ probe current and $17 \mathrm{~mm}$ working distance using halogenated dimethacrylate standards to calibrate the electron backscattering (Boyde et al., 1999) (Fig. 4). 

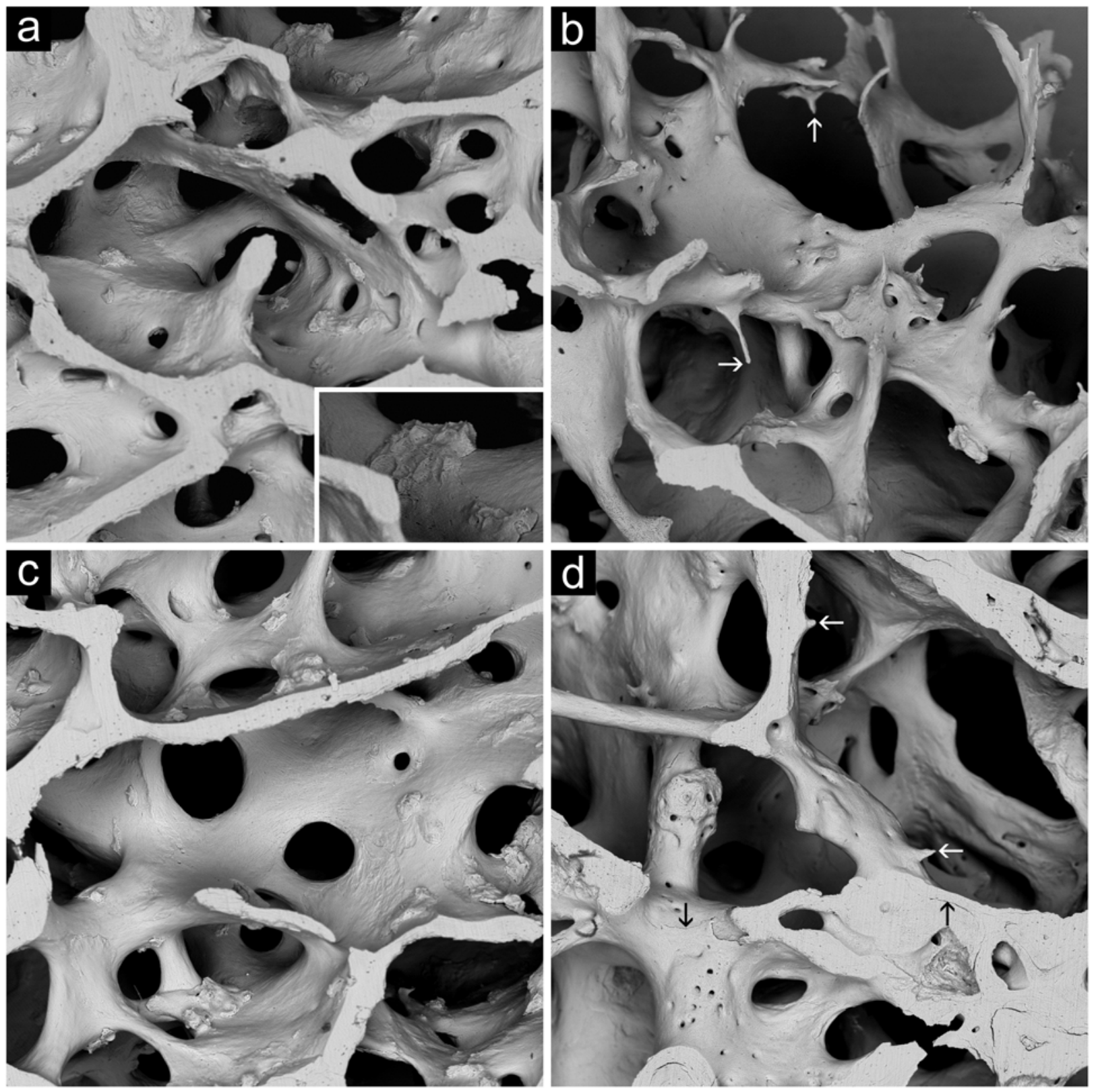

Fig. 1. $20 \mathrm{kV}$ BSE-3D-SEM images of carbon coated, macerated slice of femoral head from a 59 year old male who underwent a left total hip replacement for ochronotic osteoarthropathy. (a) Numerous excrescences project from the otherwise smooth contour of the internal bone surfaces. (Inset) High power magnification of top centre showing excrescence on pre-existing resting surface. (b) Numerous thin, free-ended (unloaded), resorbed trabeculae (arrows). (c) Another field of mostly plate-like trabeculae with numerous stucco projections from otherwise smooth surfaces. (d) Extensive microcallus patch formed from woven bone can be seen in the left hand side of the image. Several areas show incomplete integration of old and new bone packets (black arrows). Also note incomplete, resorbed trabecular ends (white arrows). All image fields (except inset of a) are $2700 \mu \mathrm{m}$ wide and high.

\section{Results}

3D topographical SEM imaging of the most severe ochronotic arthropathy sample revealed localised porotic bone within the femoral head. The more porotic regions were peripheral. In the centre of the sample, there was a greater amount of bone with fewer distinguishable trabeculae. Most of mineralised matrix was present as large bone plates joined together by short trabeculae. Close examination of the trabecular plates and rods in the regions of high bone volume revealed the presence of numerous excrescences on the surface and frequently some distinct discontinuity with the established bone surface (Fig. 1a-d).

There appeared to be various morphologies of excrescence. One very common type appears to have arisen from the incomplete resorption of something projecting above the general plane of the local bone surface, perhaps arising from partial removal of secondary or extra trabeculae, not part of the original stock of normally distributed trabeculae and which must have formed in prior marrow space. Extra trabeculae made of immature (woven) 

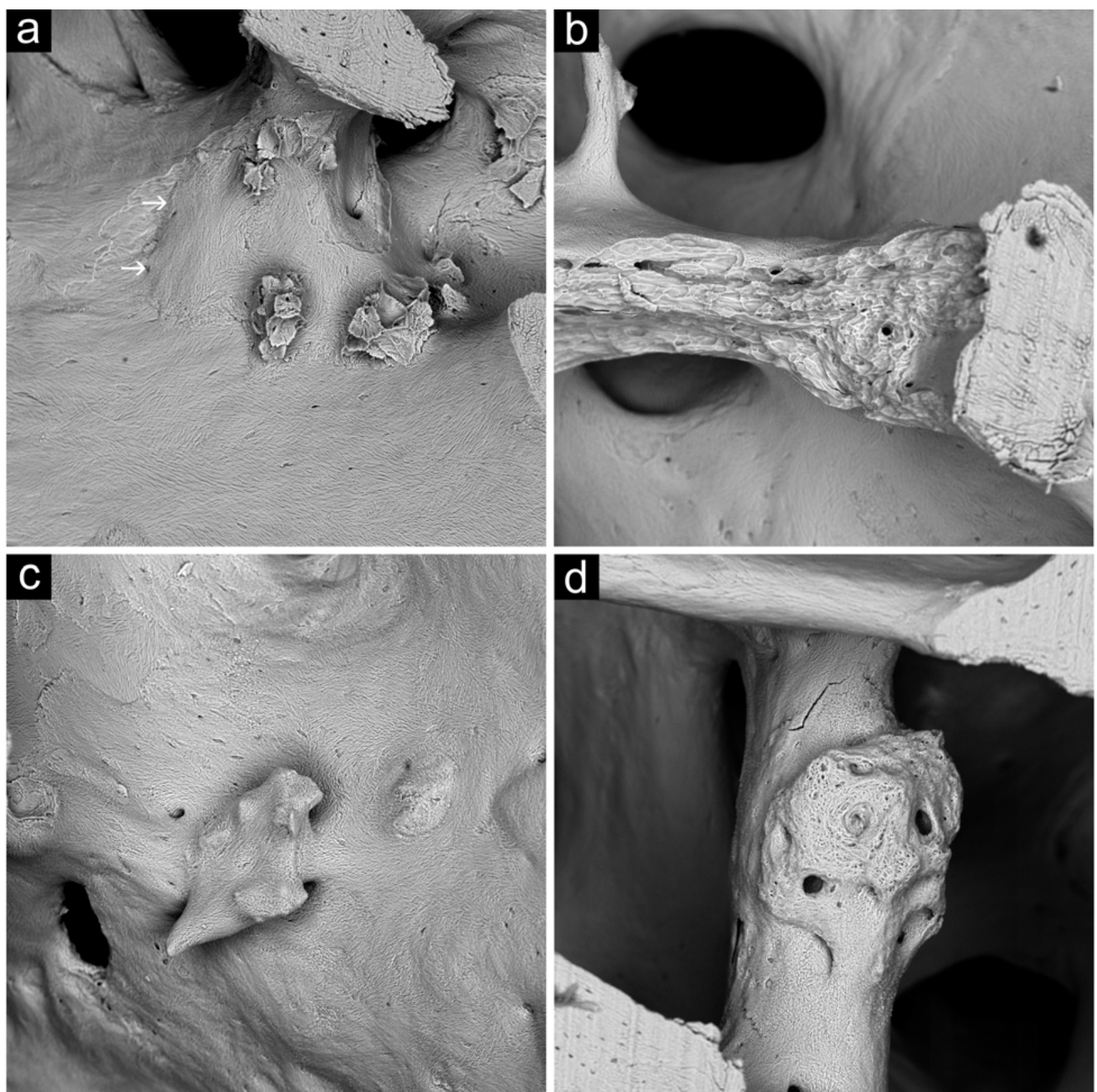

Fig. 2. Magnified 3D-SEM images from same sample observed in Fig. 1. (a) A normal resorbed area is seen at left with shallow, skimming osteoclastic resorption: this patch has been partly infilled with nearly completely mineralised bone (edge of new bone at arrows). The scene is thus typical of normal resorption-formation coupling. This contrasts strongly with the rugged scalloped edges of the obvious 'stucco' excrescences. (b) An area of normal, but unrepaired resorption on the side of a massive trabecula is seen at centre. (c) Excrescence just below centre resulted from incomplete resorption of prior trabeculae and is covered with nearly completely mineralised new bone matrix with continuous collagen fibre bundle profiles in this superficially anorganic, peroxide-treated sample (Boyde, 1972). (d) Higher magnification detail of immature bone patch seen in Fig. 1d. This microcallus patch is typical of those found in other ageing human bone samples, including in osteoporosis (Boyde, 2002, 2003). All image fields are $900 \mu \mathrm{m}$ wide and high.

bone are a common accompaniment of osteoarthritis. These are characterised by deeply scalloped surfaces and rugged edges (Fig. 1a inset and 2a). The resorption involved here is deeper and shows larger pits or scoops than those of normal resorption fields, an example of which is shown in Fig. 2b. The second appearance of excrescence is similar to the first but they have been smoothed over by new bone deposition (Fig. 2c). Neither of these types resemble the common type of microcallus patch (another form of excrescence but which we do not consider novel) found in older human trabecular bone, and which we also found in this study, an example of which is shown in Fig. 2d, which is a higher magnification of part of the field of view shown in Fig. 1d. A third appearance merges with the others, but more resembles coarse stucco and seems to have arisen where resting surfaces have been focally reactivated. These appear as random patches on the bone surface (Fig. 1a and 1c). These deposits are frequently poorly attached to the prior trabecular wall in our macerated SEM samples, as demonstrated by clearly observable discontinuities between the excrescences and the otherwise continuous trabecular surfaces. 


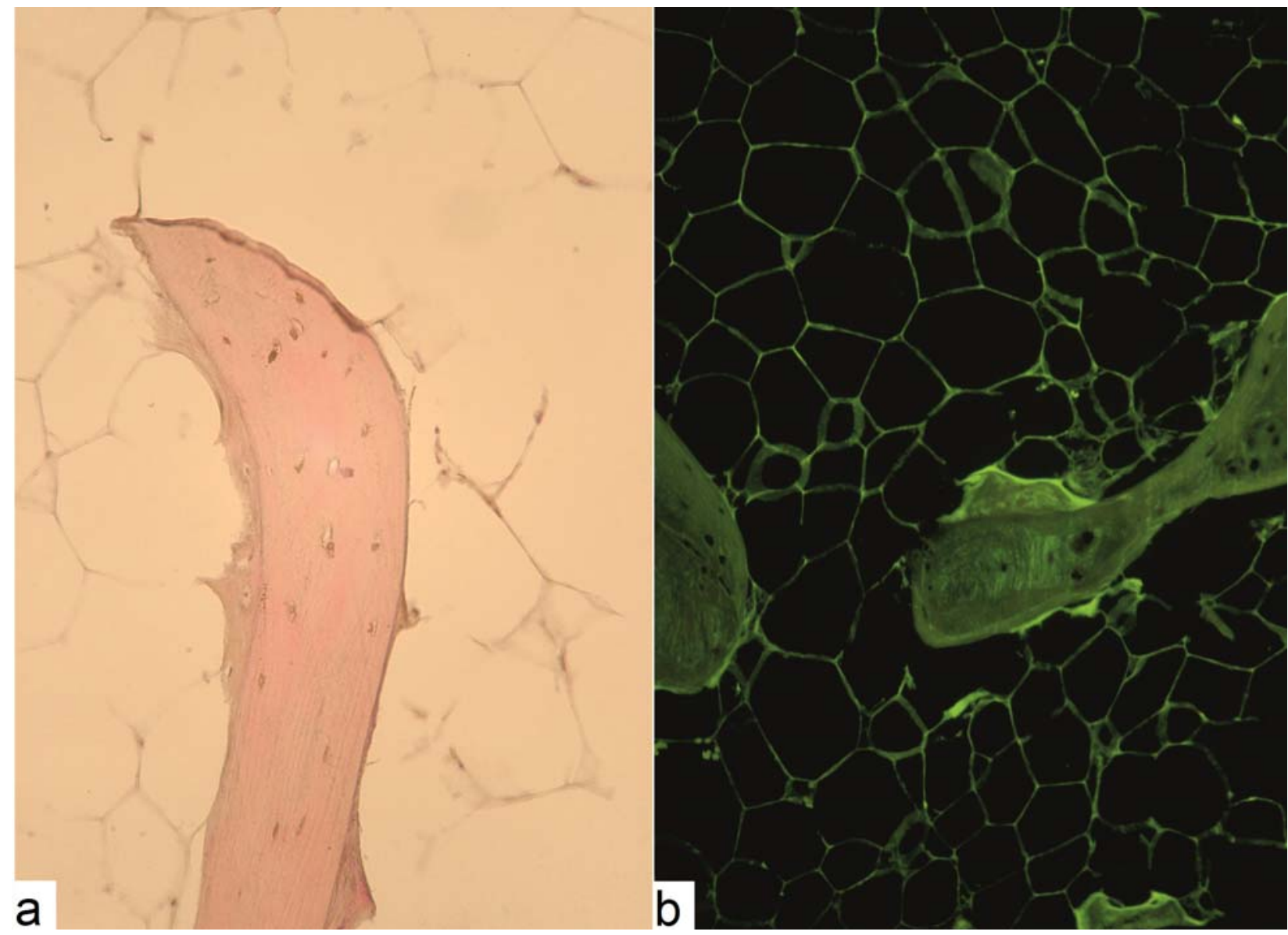

Fig. 3. Light microscopy of decalcified paraffin-embedded sections demonstrating excrescences on bone surface from same 59 year old male patient observed in Fig. 1. (a) Haematoxylin and Eosin-stained, in transmitted light, showing excrescences on both surfaces of a trabecula. Field width is $450 \mu \mathrm{m}$. (b) Epifluorescence of an unstained section under blue excitation, showing the difference in fluorescence of the established matrix and the excrescence. Field width is $600 \mu \mathrm{m}$.

Histological observations of the bone in ochronotic samples revealed multiple excrescences throughout the tissue, although the low section thickness makes it difficult to distinguish between types (Fig. 3a). Examination of AKU histological sections using autofluorescence revealed differences in the fluorescence of the established bone matrix and excrescences which were attached to their surface (Fig. 3b). qBSE-SEM imaging was performed to examine the bone quality of the AKU samples. Overall, mineralisation densities in trabeculae were within the normal range. Some of the trabecular surfaces showed surface abnormalities consistent with the 3D topographical images. Obvious excrescences showed increased mineralisation density (higher qBSE grey levels than the underlying normal mineralised bone architecture) (Fig. 4a-d). A few excrescences displayed preparative cracks at their attachment surfaces with the trabeculae, i.e., they were less well integrated with the existing bone. (Preparative cracks are seen whether analysing embedded or macerated samples, as even the most carefully dried bone dries further in the SEM vacuum giving rise to cracks which are easily recognised). Other regions, where normal new bone packets which formed over non-resorbed surfaces, may also show artefactual detachment during sample preparation for SEM, as shown in Fig. $4 \mathrm{c}$ and $\mathrm{d}$.
Examination of the osteoarthritic arthropathy samples used as reference material, with all the methods used - 3D-SEM (Fig. 5), light microscopy of sections (Fig. 6), and qBSE SEM (data not shown) - showed similar excrescences. These were present in the different forms observed in the ochronotic samples. 3D-SEM also showed that there was some poor integration of the excrescences with the pre-existing bone matrix surface. In histology, the excrescences observed were numerous "peak like" structures projecting from the surface of the trabeculae and displaying a different colour from the mineralised bone matrix when stained with $\mathrm{H}$ and $\mathrm{E}$ (Fig. $6 \mathrm{a}$ and b). At the contact surface between the existing trabeculae and the attached structure there was a distinct joint line, and the marrow space periphery of the structures also displayed rough edges, both features suggesting that integration between the excrescences and trabeculae was either not complete or occurred in an aberrant fashion.

\section{Discussion}

We report the discovery of novel microanatomical structures in bone from patients with AKU osteoarthropathy, which we have termed trabecular excrescences. We have recently 

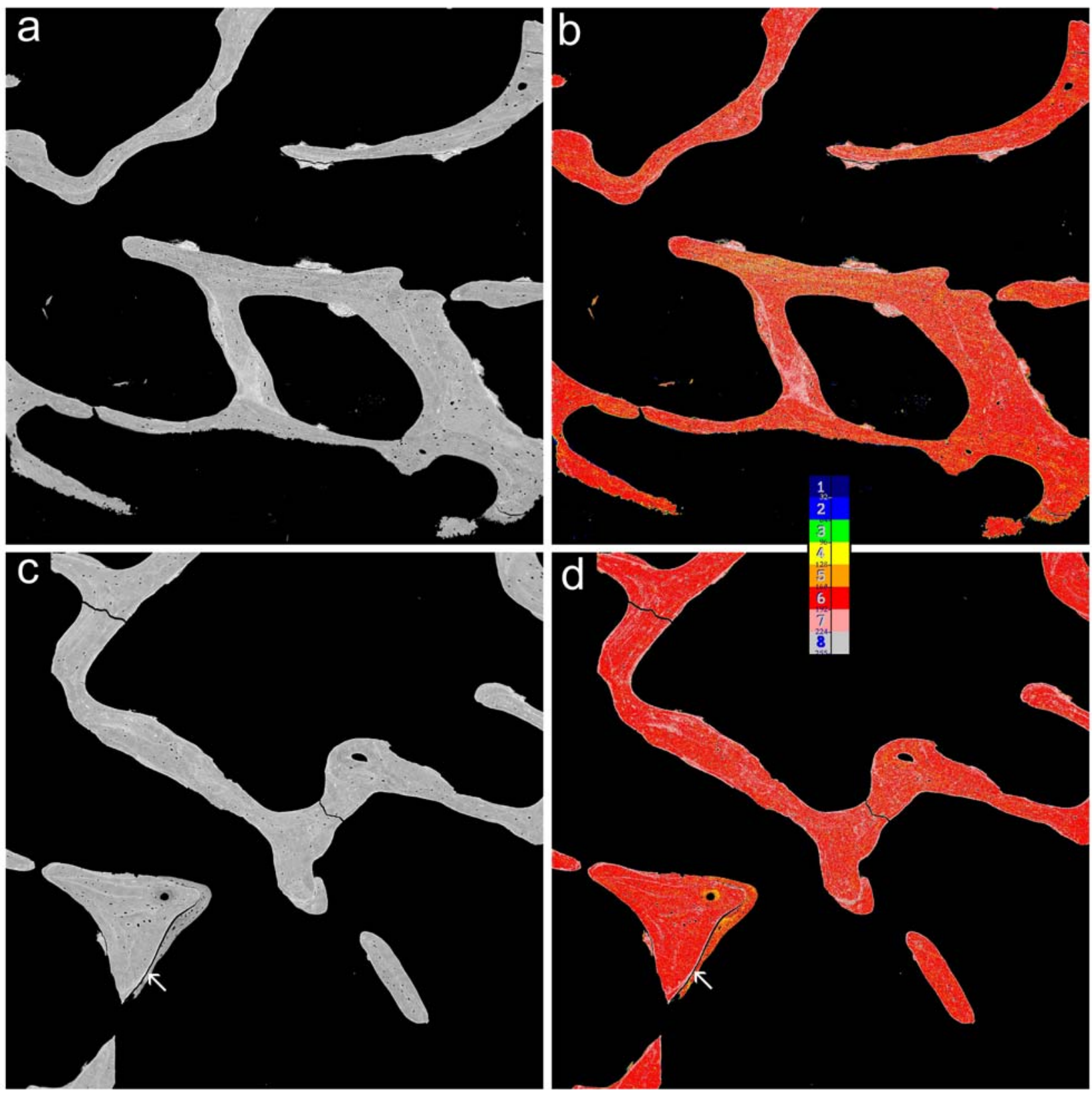

Fig. 4. $20 \mathrm{kV}$ qBSE-SEM images of polished and carbon coated PMMA-embedded slice of femoral head from same 59 year old ochronosis case: Mineralisation density was determined by reference to halogenated dimethacrylate standards. For explanation, see table in inset between panels b and $\mathbf{d}$. Grey levels were adjusted such that the value from the monobrominated standard was adjusted to zero and that for the monoiodinated standard to 255 (as detailed in Boyde et al., 1999 and Boyde and Firth, 2005). (a and b) Trabeculae show normal structure and range of mineral density. Excrescences (11 in the field of view) can be seen on the trabecular surfaces. (b) The bulk of the trabeculae exhibit normal density range, but the excrescences show hypermineralisation when compared to the trabeculae. (c and d) Trabeculae show normal architecture, with plane of poor integration between pre-existing unresorbed bone and overlying newly formed bone at lower left (arrow). All image fields are $2700 \mu \mathrm{m}$ high and wide.

demonstrated that the subchondral bone and calcified cartilage plates in AKU undergo extreme remodelling, giving them a unique phenotype (Taylor et al., 2011). Our investigation of the bone architecture revealed the presence of novel excrescences on the bone surface, originally detected by SEM analysis and subsequently confirmed using histological techniques.

Following on from this initial discovery, we then sought and discovered them in some common OA samples. To date, we have seen them in all the AKU material we have studied (12/12), but only a proportion of OA (7/10). When we only knew of their existence in AKU cases, we originally postulated that the presence of these structures may be in part related to the presence of the HGA or HGA pigment in the joint tissues of AKU patients, but the detection of these excrescences in the OA samples suggests that these structures might possibly arise due to aberrant osteoblast/osteoclast signalling, possibly brought on by abnormal loading on the joint tissues following alterations in the normal biomechanical interactions related 


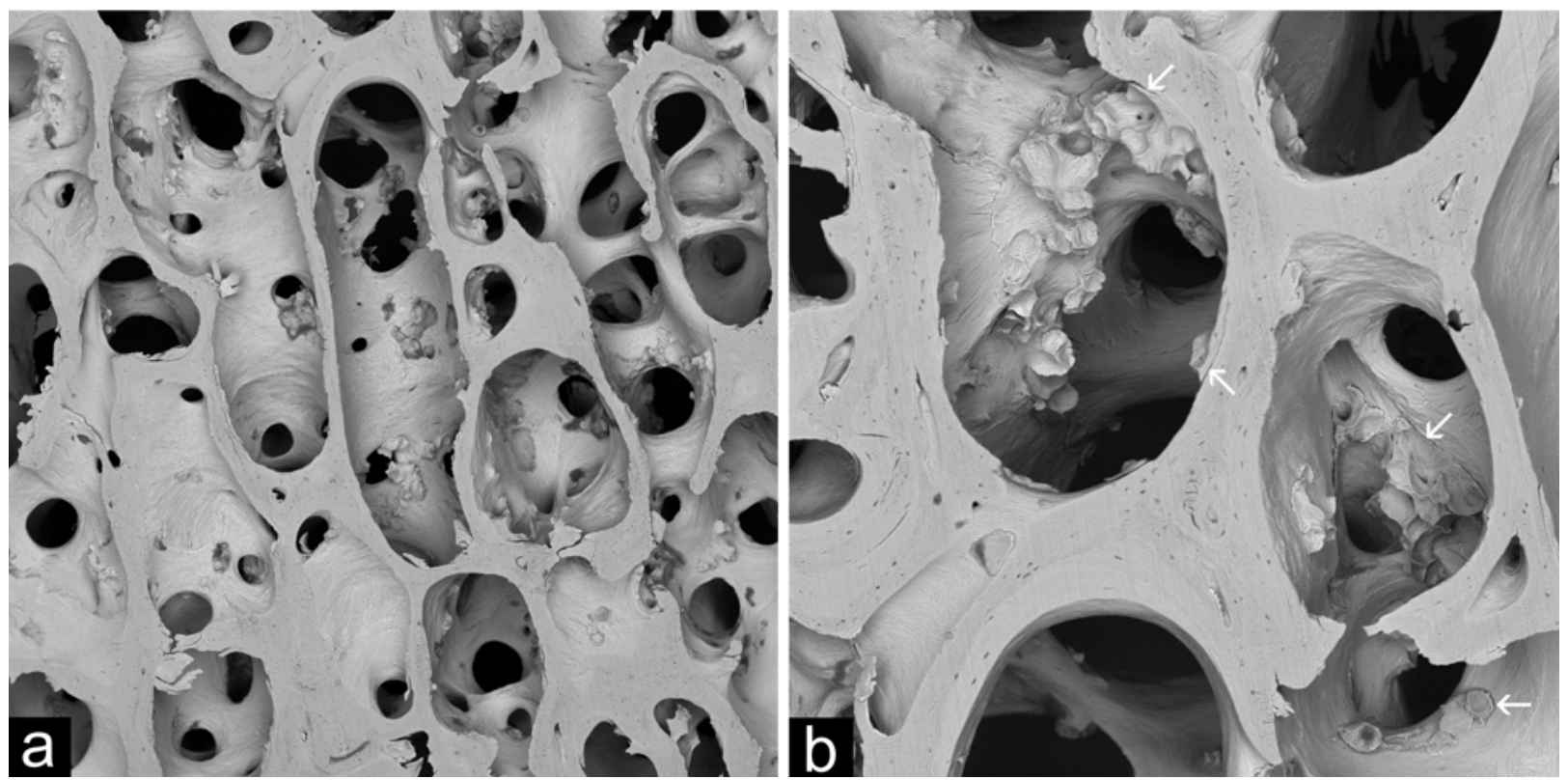

Fig. 5. 20 kV BSE-3D-SEM images of carbon-coated, macerated slice of medial femoral condyle from 51 year old female who underwent unicompartmental knee replacement for osteoarthritis. (a) Numerous excrescences can be seen on the trabeculae, predominantly with rugged edges and scalloped surfaces. Some appear darker than the surrounding bone matrix, indicative of a lower degree of mineralisation because of being more recently formed. Field width and height are $4050 \mu \mathrm{m}$. (b) Large excrescences on free bone surfaces with multiple deeply indented surfaces and intervening rugged edges can be seen. The peripheries of some of these conglomerates show a line of demarcation where they have not been well integrated with the pre-existing surfaces (arrows). Field width and height are $1780 \mu \mathrm{m}$.

to disease. Here, it should be borne in mind that the highly abnormal phenotype in AKU joint tissues appears to be at least in part a result of aberrant loading due to the presence of ochronotic pigment altering the stiffness of cartilage (Taylor et al., 2011). The presence of these excrescences within some common OA samples would then be further evidence that the role of abnormal loading across the joint in OA patients is significant in the onset and progression of OA (Griffin and Guilak, 2005).

The relationship between bone and cartilage in the progression of joint failure has been well investigated, and it is generally accepted that there are key roles for both in both the commencement and the progression of joint degeneration, particularly in OA (Burr, 2004). It is clear that the basic descriptions of changes involved in joint degeneration are not limited to bone and cartilage, but are more appropriately attributed to highly specialised sub-divisions of these tissues which are related to their homeostatic and biomechanical functions.

The detection of these 3 types of excrescence, which may or may not all be interrelated, may be indicative of altered phenotype of cells and the abnormal homeostasis and signalling in these tissues. One type of excrescence arises from incomplete resorption of branching trabeculae, which do not appear to be part and parcel of the 'normal', pre-existing trabecular architecture. It is common in $\mathrm{OA}$ for marrow spaces to be filled with immature 'woven' bone, which some might describe as 'micro'callus, but the term callus should not be taken to imply that the tissue is formed in response to overt fracture. Similar obliteration of marrow space occurs in the densification of normal bone in response to heavy impact exercise with no evidence of any sign of trabecular microfracture (Boyde and Firth, 2005). Modelling of this excess bone tissue to restore the homeostatic mechanical loading balance might then result in this appearance.

However, these structures appear to be closely related to the another type of excrescence, in which short, branched trabeculae that had been subjected to partial osteoclastic action have been smoothed over by the deposition of new layers of bone by osteoblasts. The deposition of new matrix on these structures leads to smooth partial trabeculae projecting into the marrow space. The functional contribution of these partial trabeculae is questionable given that without connections at both ends, they could not provide any form of structural support in biomechanical function.

The type of excrescence which appears as coarse stucco represents spots stuck on resting surfaces that have been focally reactivated. In turn, their surface morphology appears very frequently also to be altered by subsequent resorption. The coupling between bone resorption and formation is supposedly tightly regulated in the normal mature young adult, such that bone is only removed if there is going to be new bone to replace it. The generally accepted view is that osteoblasts normally arrive at the sites of resorption recently vacated by osteoclasts (Parfitt, 2000). However, this coupling phenomenon is not the dominant form of turnover in the growing and drifting skeleton (Enlow, 1963), and is less tight in ageing when trabecular modelling occurs (Boyde, 2002). It can also be severely unbalanced in pathological states to cause bone loss (osteoporosis) or deposition (sclerosis). The deposition of new bone on the surface of existing trabeculae without 


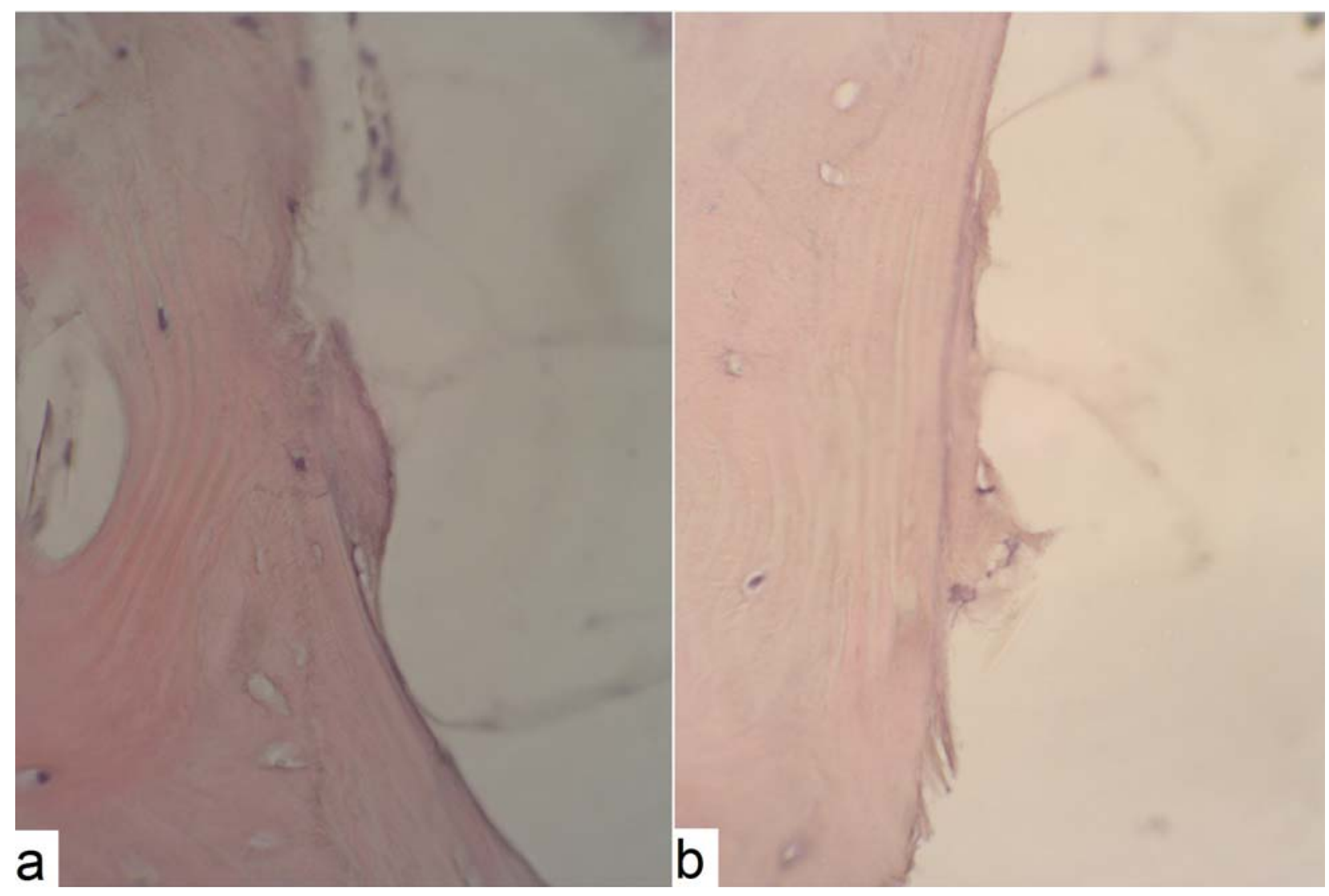

Fig. 6. $\mathrm{H}$ and E-stained decalcified paraffin-embedded sections. (a) 51 year old female knee OA case, (b) 74 year old male knee OA case. Both sections demonstrate the presence of excrescences on the trabecular bone surface. Both field widths are $300 \mu \mathrm{m}$.

osteoclastic resorption suggests that such coupling does not exist in this case. These structures also appear to lack the ordered lamellar structure typically observed in bone.

Whilst the tissues examined in this study are from two different diseases, they both display similar structures on the trabecular network. The advanced osteoarthropathy of AKU provides an unprecedented opportunity to investigate regional unloading and factors that might be involved in this process. The authors made the initial discovery working on AKU samples, where the novel microanatomical structures which we group as excrescences were identified easily on internal surfaces. The follow up observations of OA samples as reference material for this study, and the catalogue of OA samples that the authors have observed previously, demonstrated that these structures are present, but are not as obvious or widespread. This observation in OA reference material proves that the expression of HGA by ochronotic cells would not play a factor in the presence of these novel structures (Laschi et al, 2011). This may suggest that whatever mechanisms are involved in their formation are more severe in the pathology of AKU. It has been demonstrated that there are significant changes in the mechanical properties of alkaptonuric cartilage compared to OA and normal (Taylor et al, 2011). The dramatic biochemical and mechanical changes seen in AKU cartilage will cause alterations in how the load is distributed across the underlying bone and may in part be the reason that causes a more widespread presence of these structures in AKU over OA; 12/12 vs. 7/10. Typically,
AKU patients present for joint replacement earlier than the OA references with a more severe OA. The mean age of AKU patients in the study was at least 4 years less than their OA controls. It may be that the 3 OA samples which did not show excrescences may at some point later in life have gone on to develop them.

In summary, through meticulous examination using a combination of SEM and light microscopy, we have identified novel microanatomical structures in bone. These were first identified in the osteoarthropathy of $\mathrm{AKU}$, where they are abundant, and subsequently in OA, where they proved more difficult to find. Their size and distribution would make these excrescences difficult to find using other imaging modalities, including microCT - though with the benefit of hindsight we have been able to find them in microCT reconstructions where we knew they were present from prior SEM study. Much further investigation is required to determine their development and functional significance and whether they are present in other pathological conditions.

\section{Acknowledgements}

The authors would like to express our deep gratitude to the patients who kindly agreed to the use of their tissue for this study, and our thanks to Mo Arora who provided excellent technical assistance. We would also like to thank the Alkaptonuria Society (AT), Rosetrees Trust (AT), the 
Bone Research Society (AT), the UK National Lottery, and the Horserace Betting Levy Board (AB) for funding. All authors have no conflicts of interest. The authors confirm that there are no known conflicts of interest associated with this publication and there has been no significant financial support for this work that could have influenced its outcome.

\section{References}

Bálint G, Szebenyi B (2000) Hereditary disorders mimicking and/or causing premature osteoarthritis. Baillieres Best Pract Res Clin Rheumatol 14:219-250.

Boyde A (1972) Scanning electron microscope studies of bone. In: Bourne GH (ed) The Biochemistry and Physiology of Bone, 2nd ed., Vol. 1. Academic Press, New York, pp 259-310.

Boyde A. (2002) Morphologic detail of aging bone in human vertebrae. Endocrine 17: 5-14.

Boyde A (2003) The real response of bone to exercise. J Anat 203: 173-189

Boyde A, Firth EC (2005) Musculoskeletal responses of 2-year-old thoroughbred horses to early training. 8 . Quantitative back-scattered electron scanning electron microscopy and confocal fluorescence microscopy of the epiphysis of the third metacarpal bone. N Z Vet J 53: 123132.

Boyde A, Travers R, Glorieux FH, Jones SJ (1999) The mineralisation density of iliac crest bone from children with osteogenesis imperfecta. Calcif Tissue Int 64: 185-190.

Burr DB (2004) Anatomy and physiology of the mineralized tissues: Role in the pathogenesis of osteoarthrosis. Osteoarthritis Cartilage 12: S20-S30.

Di Franco M, Coari G, Bonucci E (2000) A morphological study of bone and articular cartilage in ochronosis. Virchows Arch 436: 74-81.

Enlow DH (1963) Principles of bone remodelling: An account of post-natal growth and remodeling processes in long bones and the mandible. Charles Thomas, Springfield.

Ferguson VL, Bushby AJ, Boyde A (2003) Nanomechanical properties and mineral concentration in articular calcified cartilage and subchondral bone. J Anat 203: 191-202.

Flachsmann R, Broom ND, Hardy AE, Moltschaniwskyj G (2000) Why is the adolescent joint particularly susceptible to osteochondral shear fracture? Clin Orthop Relat Res 381: 212-221.

Frost HM (1964) Dynamics of bone remodelling. In: Frost HM (ed.) Bone biodynamics, Little Brown, Boston, pp 315-333.

Frost HM (1991) A new direction for osteoporosis research: A review and proposal. Bone 12: 429-437.

Gray C, Boyde A, Jones SJ (1996) Topographically induced bone formation in vitro: implications for bone implants and bone grafts. Bone 18: 115-123.

Griffin TM, Guilak F (2005) The role of mechanical loading in the onset and progression of osteoarthritis. Exerc Sport Sci Rev 33: 195-200.

Hill PA. (1998) Bone remodelling. Br J Orthod 25: 101-107.
Jayasinghe JA, Jones SJ, Boyde A (1993) Scanning electron microscopy of human lumbar vertebral trabecular bone surfaces. Virchows Arch A Pathol Anat Histopathol 422: $25-34$.

Jayasinghe JA, Jones SJ, Boyde A (1994) Threedimensional photographic study of cancellous bone in human fourth lumbar vertebral bodies. Anat Embryol (Berl) 189:259-274.

Kraus VB (2011) Rare osteoarthritis: Ochronosis, Kashin-Beck disease and Mseleni joint disease. In: Hochberg MC, Silman AJ, Smolen JS, Weinblatt ME, Weisman MH (eds) Rheumatology, 5th ed. Mosby, Philadelphia, pp 1825-1837.

Lagier, R (2006) Ochronotic arthropathy, an approach to osteoarthritis bone remodelling. Rheumatol Int 26: 561 564.

Laschi M, Tinti L, Braconi D, Millucci L, Ghezzi L, Amato L, Selvi E, Spreafico A, Bernardini G, Santucci A (2011). Homogentisate 1,2 dioxygenase is expressed in human osteoarticular cells: Implications in alkaptonuria. J Cell Physiol doi: 10.1002/jcp.24018.

Parfitt AM (1982) The coupling of bone formation to bone resorption: a critical analysis of the concept and of its relevance to the pathogenesis of osteoporosis. Metab Bone Dis Relat Res 4: 1-6.

Parfitt AM (2000) The mechanism of coupling: a role for the vasculature. Bone 26: 319-323.

Russell RGG. (2003) Pathogenesis of osteoporosis. In: Hochberg MC, Silman AJ, Smolen JS, Weinblatt ME, Weisman MH (ed) Rheumatology, 3rd ed. Mosby, New York, pp 2075-2147.

Taylor AM, Wlodarski B, Prior IA, Wilson PJM, Jarvis JC, Ranganath LR, Gallagher JA (2010) Ultrastructural examination of tissue in a patient with alkaptonuric arthropathy reveals a distinct pattern of binding of ochronotic pigment. Rheumatology (Oxford) 49: 14121414.

Taylor AM, Boyde A, Wilson PJM, Jarvis JC, Davidson JS, Hunt JA, Ranganath LR, Gallagher JA (2011) The role of calcified cartilage and subchondral bone in the initiation and progression of ochronotic arthropathy in alkaptonuria. Arthritis Rheum 63: 3887-3896.

\section{Discussion with Reviewers}

Reviewer I: Given the small sample size it is likely not possible to definitely evaluate for age-related associations, but given the samples available, is there any apparent increase in these excrescences with age?

Authors: The reviewer raises an interesting question. Age does not seem to be the determining factor with regards to presence of, or increasing number of, excrescences. It would appear that disease type is more associated with excrescences: 12/12 AKU samples demonstrated excrescences and only 7/10 OA. We can only speculate as to whether the $3 \mathrm{OA}$ cases which did not display excrescences would have gone on to do so.

Reviewer I: Are there any discernible patterns or clustering of excrescences, i.e. are they localised to particular regions of the bone or distance from the calcified cartilage? 
Authors: We did not note any patterns with regards to the presence of excrescences. Excrescences were present on the bone side of the subchondral plate in samples where it was present, on trabeculae directly supporting the subchondral plate, and attached to trabeculae that were distant to the subchondral plate.

Reviewer II: It would be interesting to see the quantification of the micro-structural phenotypes: incidence and surface extent. Is there a quantifiable difference between AKU and OA in terms of the micro-structural phenotypes? The authors indicate that only some OA specimens show the phenotypes of AKU and to a lesser extent. Do all the patients exhibit the same degree of severity of either AKU or OA?

Authors: We have made no attempt to quantify these structures by surface extent, as the incidental discovery was so striking. By patient number, $100 \%$ of AKU samples analysed show the excrescence phenotype, whereas only $70 \%$ of OA samples present with excrescences. One of our hypotheses is that abnormal loading in the disease state is involved; AKU cartilage and subchondral bone are highly abnormal (Taylor et al., 2011), even more so than the structural and molecular changes observed in $\mathrm{OA}$ cartilage and subchondral bone. Within each disease type there is a widespread variation in presence of excrescences, with some samples having much more easily identifiable and numerous excrescences compared to other samples. However, we do not yet have a reliable means to quantify these structures.

Reviewer II: Do incidence and extent of phenotypes depend on the age and sex of patient? The number of specimens seems adequate to support such analysis.
Authors: The purpose of this work is to alert the bone and cartilage community to a stunning discovery initially made by morphological SEM study in cases of ochronotic arthritic change, later expanded to cases of OA without $\mathrm{AKU}$, and who knows whether some examples of this will eventually be put down to 'ageing' - though the latter conclusion would always be arguable because any aged person is likely to have 'OA'. We do not yet have enough cases to provide meaningful 'demography'. We have ample evidence for a new phenomenon of undoubted significance, and we hope that the field will be the better for this knowledge.

Reviewer III: Since Marcella Laschi has shown that connective tissue cells express homogentisate dioxygenase (Laschi et al., 2011), is it likely that the excrescences are formed by cells that are preferentially forming the melanin polymer?

Authors: We can state that this is not the case given that these structures are found in OA "reference" samples, where any effect that may be produced in alkaptonuric tissue is completely absent. The authors speculate that the excrescences are a result of aberrant osteoblast/osteoclast signalling, although what signalling pathway is involved in the formation of these structures is not clear, but would warrant a future investigation.

Reviewer III: Is there any evidence to indicate that the pigment preferentially blocks stem cell differentiation along the osteoblast lineage or alters collagen synthesis and mineralisation?

Authors: We have no evidence to support or refute any involvement of melanin pigment in the blocking of stem cell differentiation along the osteoblast lineage. 